\title{
Uma análise percentual dos artigos de periódicos publicados pelos docentes de fonoaudiologia da Universidade São Paulo
}

\section{Percentage analysis of scientific productivity of speech, language \& hearing science professors at USP}

Rondinelli D. Herculano'1, Ana Maria Q. Norberto²

\begin{abstract}
RESUMO
Objetivo: O objetivo do presente trabalho foi analisar/quantificar a produtividade científica dos docentes pertencentes aos três cursos de graduação em Fonoaudiologia ministrados pelas: 1-) Faculdade de Medicina de Ribeirão Preto (FMRP), 2-) Faculdade de Odontologia de Bauru (FOB), 3-) Faculdade de Medicina (FM). Estas três faculdades são unidades da Universidade de São Paulo (USP). Métodos: Para cada professor, analisamos o número total de artigos (na plataforma Lattes), o título e indexação destes periódicos. Resultados: Uma análise percentual mostrou o perfil de publicação de cada faculdade e a relevância destes artigos. Observamos que os docentes pertencentes à FMRP tiveram os artigos publicados restritos a apenas 6 revistas Estas revistas corresponderam a 50\% dos artigos publicados por estes docentes. Na FOB, observamos uma menor concentração de artigos publicados, dos quais $62 \%$ dos artigos publicados foram distribuídos em 11 revistas. Entretanto, 30\% destes artigos se concentraram na revista Pró-Fono, Revista Brasileira de Otorrinolaringologia e na Revista da Sociedade Brasileira de Fonoaudiologia. Neste grupo de docentes, observou-se uma quantidade ligeiramente maior de artigos indexados no ISI. Sobre os docentes pertencentes a FM, foi observada uma homogeneidade similar aos docentes pertencentes à FOB, ou seja, $72 \%$ dos artigos publicados pelos docentes da FM estão concentrados em 10 revistas. Entretanto, observamos que $40 \%$ dos artigos publicados por estes docentes se concentram na revista Pró-Fono e na Revista da Sociedade Brasileira de Fonoaudiologia. Conclusão: Observamos uma grande concentração de publicação em poucas revistas nas três unidades da USP. Estes docentes publicaram poucos artigos em revistas indexados ao ISI.
\end{abstract}

Palavras-chave: Índice de Periódicos. Indicadores de Produção Científica. Indicadores Bibliométricos.

1. Pós-Doutorando do Instituto de Física de São Carlos-USP. Departamento de Física e Ciências dos Materiais, IFSC, Universidade de São Paulo, São Carlos (Brasil).

2. Fonoaudióloga, Mestranda em Neurociências pelo Departamento de Neurociências e Ciências do Comportamento, FMRP, Universidade de São Paulo, Ribeirão Preto (Brasil).

Pesquisa financiada pela: CAPES, FAEPA e CNPq.
Correspondência: Rondinelli Donizetti Herculano Pós-Doutorando do Departamento de Física e Ciência dos Materiais, IFSC, USP Trabalhador Sãocarlense, 400 - Grupo de Polímeros, 13560970, São Carlos, SP Brasil Fax: +55-16-3373-9797 Ramal: 234 E-mail: rond@ursa.ifsc.usp.br

Artigo recebido em 27/03/2009 Aprovado em 31/08/2009 


\section{Introdução}

O fluxo da comunicação científica inclui a publicação formal de resultados de pesquisa, a recuperação de informação, o acesso à literatura publicada e a comunicação informal e de intercâmbio entre pesquisadores. É um fluxo contínuo, pois conhecimentos publicados e assimilados dão origem a novos conhecimentos, pesquisas e publicações, regidos por uma dinâmica específica e influenciados pelas relações com a sociedade. ${ }^{1}$ A atividade científica é descrita por Pellegrini Filho ${ }^{2}$ em três processos básicos: produção (pesquisa), circulação e incorporação do conhecimento. $\mathrm{O}$ autor destaca que esses processos "estão imersos em uma totalidade social a qual influenciam, e que por sua vez, também são influenciados, principalmente através de instâncias mediadoras como as de financiamento, formação de recursos humanos, legitimação, legislação e normas, canais de disseminação e comunicação de informação, estrutura dos sistemas de saúde, etc".

De acordo com Price $(1976)^{3}$, os indicadores cienciométricos podem ajudar a mensurar a contribuição dada por cada país ao acervo mundial de pesquisa, além de localizar geograficamente as pesquisas por autor, assunto e acompanhar o crescimento em cada área da ciência.

Uma forma de verificar a quantidade e/ou título de periódicos é por meio de base (banco) de dados. No Brasil, há o sistema de currículos chamado Platafoma Lattes. ${ }^{4}$ Este banco de dados inclui currículos dos pesquisadores/professores pertencentes às instituições acadêmicas brasileiras. ${ }^{4}$ A plataforma Lattes possui cerca de 850 mil currículos, sendo que $30 \%$ destes são de mestres e doutores e 38\% de graduados e estudantes de graduação, que pertencem à cerca de 4000 diferentes instituições de pesquisa registradas no sistema.
Além da plataforma Lattes, existem renomados bancos de dados internacionais, tais como: Thompson ISI Web of Science ${ }^{5}$ e o SCOPUS. ${ }^{6}$

A base de dados ISI7 representa um conjunto de periódicos que constituem uma literatura influente internacionalmente. Representa a porção de pesquisa que é publicada e citada na literatura da elite internacional. Além disso, geralmente representa a melhor ciência realizada em qualquer nação. No entanto, sabese que pesquisas de ponta são também publicadas em periódicos locais ou regionais não indexados no ISI.

Recentemente, o "SCOPUS"6 que é o maior banco de dados de resumos e citações com cerca de 17 mil revistas, surgiu para competir com Thompson ISI Web of Science. A cobertura do ISI é ainda um grande problema, principalmente porque somente uma pequena porcentagem $(5 \%)$ dos artigos publicados no mundo está incluída no ISI, ou seja, cerca de 7 mil títulos. A cobertura do SCOPUS não pára de crescer, pois recentemente foi incorporada à sua base de dados o $\mathrm{SciELO}^{8}$ que possui em seu portifólio 473 periódicos de 12 países ibero-americanos e 1 caribenho.

Tais bancos de dados possuem informações (currículos e artigos de periódicos) de várias ciências, dentre elas a fonoaudiologia. Uma ciência recente, vinda ao Brasil na década de 50. Voltada inicialmente ao contexto educacional e com procedimentos atuais nas áreas de linguagem, motricidade orofacial, audiologia, voz e mais recentemente em saúde coletiva. Além disso, a fonoaudiologia busca conhecimentos nas áreas de lingüística, psicologia, sociologia, biologia e física. ${ }^{9}$

Pelo exposto, o presente trabalho analisou a participação dos docentes dos cursos de fonoaudiologia da Universidade de São Paulo nos referidos bancos de dados, por meio da publicação de artigos. Na Tabela 1, são apresentados os departamentos nos quais os cursos de fonoaudiologia da USP são ministrados, além da sua data de criação.

\section{Tabela 1}

Cursos de graduação em fonoaudiologia da Universidade de São Paulo.

\begin{tabular}{lllc}
\hline Unidade & Departamento & $\begin{array}{c}\text { Data de criação } \\
\text { do Departamento }\end{array}$ & $\begin{array}{c}\text { Início do curso } \\
\text { de Fonoaudiologia }\end{array}$ \\
\hline FMRP & $\begin{array}{l}\text { Oftalmologia, Otorrinolaringologia e } \\
\text { Cirurgia de Cabeça e Pescoço }\end{array}$ & Abril de 1959 & 2003 \\
FM & Fisioterapia, Fonoaudiologia e Terapia Ocupacional & Dezembro de 1999 & 1958 \\
FOB & Fonoaudiologia & Maio de 1982 & 1990 \\
\hline
\end{tabular}


Recentemente, alguns trabalhos relacionados à cienciometria foram publicados. Entretanto, não existem trabalhos detalhados referentes à publicação de periódicos em fonoaudiologia. Considerando a importância da produção cientifica para as agências de fomento para liberação de verbas, cargos administrativos dentro das universidades, dentre outros, analisamos o perfil de publicação dos docentes pertencentes aos cursos de fonoaudiologia da USP.

\section{Métodos}

A coleta de dados foi realizada na internet, entre janeiro e fevereiro de 2009. Primeiramente, houve o acesso ao site das três unidades da USP que oferecem os cursos de graduação em fonoaudiologia, ou seja, a Faculdade de Medicina (FM), a Faculdade de Medicina de Ribeirão Preto (FMRP) e a Faculdade de Odontologia de Bauru (FOB). Nesta etapa, foi realizada a coleta de dados relativa ao total de docentes pertencentes aos cursos de graduação em fonoaudiologia destas unidades.

As informações relacionadas à quantidade de artigos e aos títulos de periódicos publicados pelos docentes pertencentes as três unidades da USP foram obtidas com base nas informações contidas no Currículo Lattes de cada docente. (CL). A busca foi realizada acessando a "Plataforma Lattes" no campo "busca pesquisadores" e foi empregado o modo de busca simples. Foi digitado o nome completo de cada docente pertencente a cada uma das unidades participantes desta pesquisa (FM, FMRP e FOB).

A partir dos dados obtidos pelo currículo Lattes, acessou-se "Thompson ISI Web of Science" no campo "Advanced Search" para verificar se os periódicos publicados pelos docentes das unidades analisadas estão indexados no ISI.

Para uma análise da relação tipo de publicação/índice-h, calculamos o mesmo utilizando a fórmula de Hirsch publicada em $2005^{10}$, na qual estabelece que $\mathrm{h}$ seja definido como o número de artigos publicados por um pesquisador, com citações maiores ou iguais a esse número. Este método tem a vantagem de avaliar a produção científica de pesquisadores porque combina produtividade e o impacto da pesquisa.

\section{Resultados}

O principal objetivo deste trabalho foi analisar os artigos de periódicos publicados pelos docentes dos cursos de fonoaudiologia da FOB, FMRP e FM. Na análise dos dados foi possível observar que os docentes da FMRP publicam a maior parte de seus artigos em seis revistas (Figura 1). Os sete docentes do curso de fonoaudiologia pertencentes à FMRP publicaram mais artigos em periódicos não indexados no ISI, ou seja, em artigos regionais e locais, tais como: Jornal Brasileiro de Fonoaudiologia, Revista da Sociedade Brasileira de Fonoaudiologia, Pró-Fono e Revista Brasileira de Otorrinolaringologia.

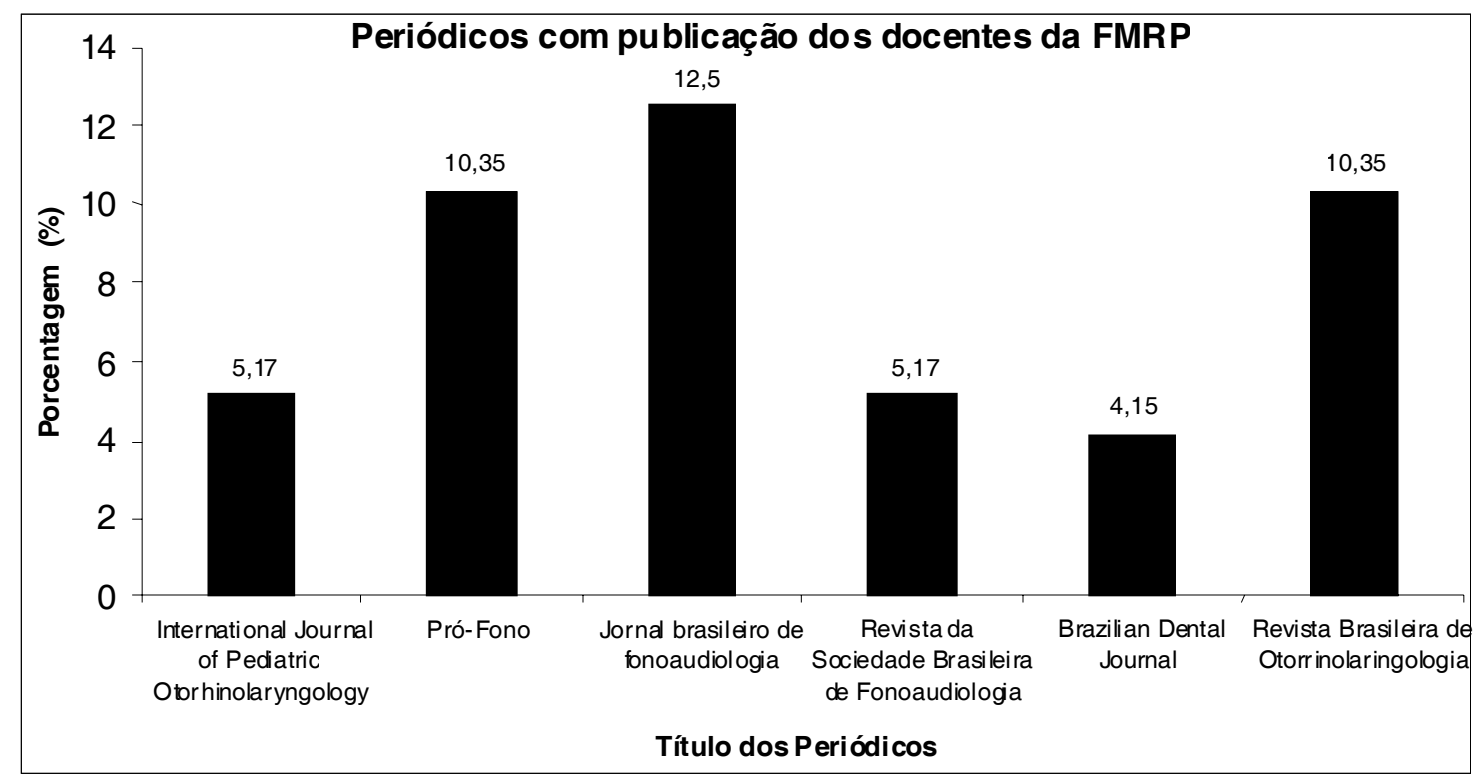

Figura 1. Percentagem dos títulos de artigos de periódicos publicados pelos docentes vinculados a FMRP. 
Os 24 docentes do curso de fonoaudiologia pertencentes à FOB também publicaram mais artigos em periódicos não indexados no ISI, tais como: Revista Brasileira de Fonoaudiologia, Pró-Fono, Revista de Distúrbios da Comunicação e Revista Brasileira de Otorrinilaringologia.

Observou-se também que $62 \%$ dos artigos publicados pelos docentes da FOB estão restritos a 11 revistas (Figura 2). Cerca de 15\% dos artigos publicados pelos docentes da FOB estão na revista PróFono, enquanto que 8,85\% estão na Revista Brasileira de Otorrinolaringologia e outros 8,25\% estão publicados na Revista Brasileira de Fonoaudiologia. Neste caso, apenas estas três revistas são responsáveis por mais de $30 \%$ dos artigos publicados pelos docentes dessa unidade.

Com relação à análise de dados relativa ao número de artigos presentes na plataforma Lattes dos 13 docentes de fonoaudiologia da FM, foi observado que $72 \%$ dos artigos publicados por eles estão concentrados em 10 revistas. A Figura 3 mostra que mais de $40 \%$ dos artigos publicados por estes docentes se concentram em duas revistas: Pró-Fono e Revista Brasileira de Fonoaudiologia. Vale ressaltar que estas 10 revistas não fazem parte da plataforma ISI Web of Science.
Um dado importante é que as pesquisas (número de artigos e projetos) não estão atreladas apenas a cursos de graduação. Deste modo, consideramos se as três unidades da USP estudadas possuem programas de pós-graduação e se os professores analisados estão inseridos nestes programas. Assim, com o auxílio da plataforma Lattes, observamos que apenas 4 dos 7 docentes pertencentes à FMRP são ligados a 1 programa de pós-graduação, enquanto 12 docentes pertencentes à FM são ligados a 8 programas de pós-graduação. Já em relação à FOB, 14 docentes são ligados a 18 programas de pós-graduação. A Tabela 2 mostra o número de docentes pertencentes aos programas de pós-graduação e o número de artigos analisados.

Para uma análise mais crítica dos dados, foi calculado a média de artigos na Plataforma Lattes, artigos indexados no ISI Thompson Web of Science e o índice-h de todos os docentes analisados neste trabalho. A Tabela 3 apresenta a média dos indices-h, artigos indexados no ISI e artigos na plataforma Lattes.

\section{Discussão}

Diferenças entre as publicações dos professores da FMRP, FOB e FM vinculados aos cursos de

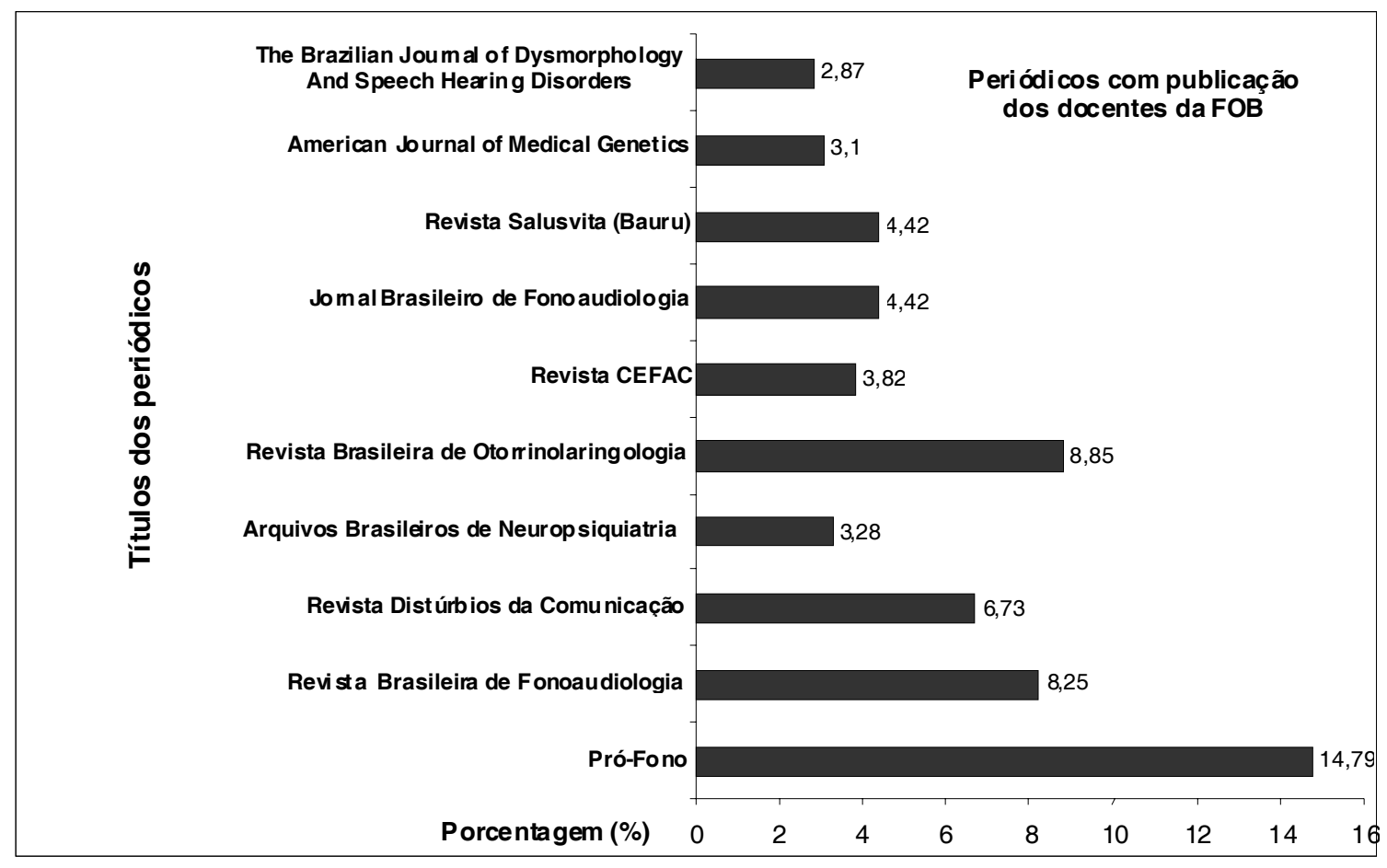

Figura 2. Percentagem dos títulos de artigos de periódicos publicados pelos docentes vinculados a FOB. 


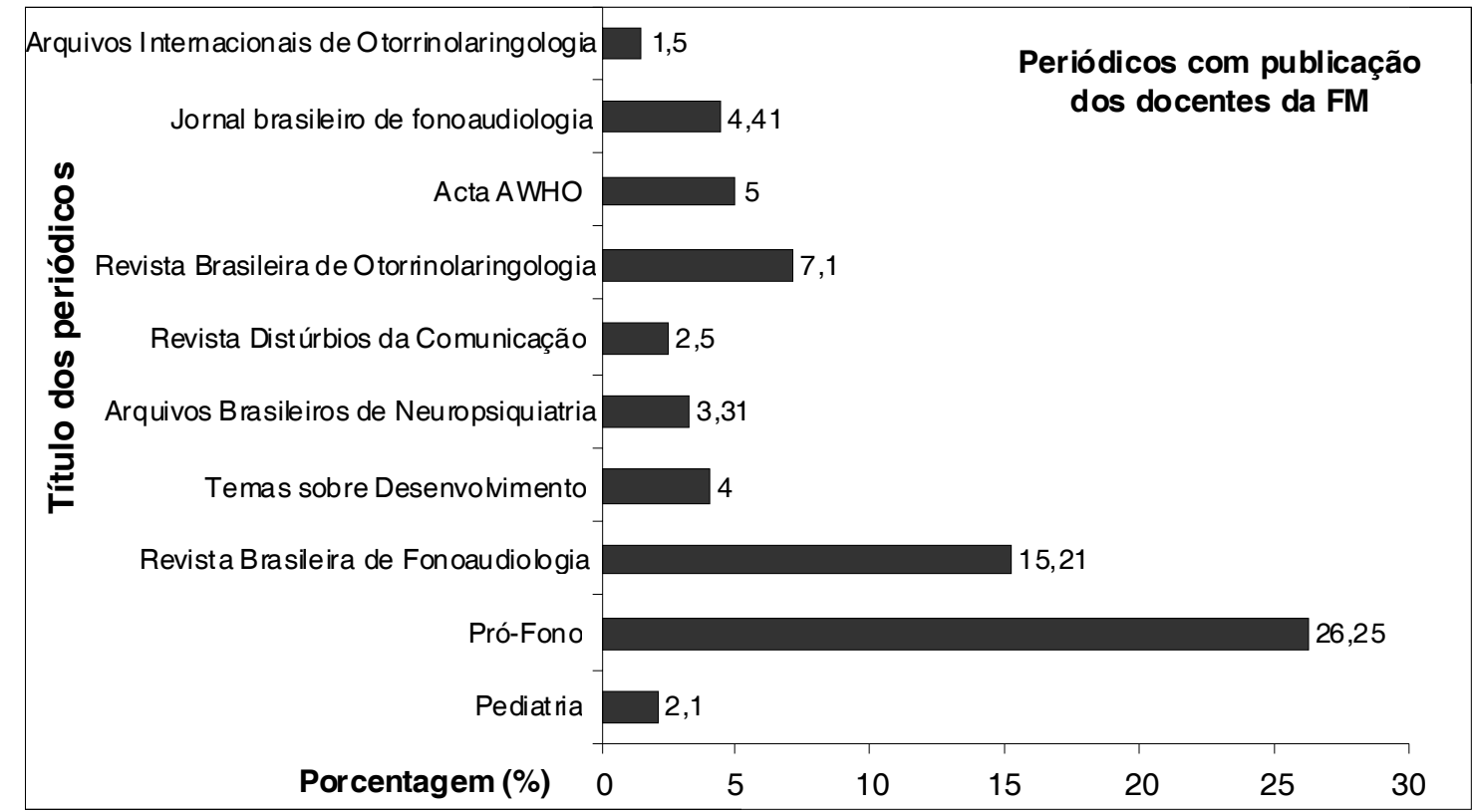

Figura 3. Percentagem dos títulos de artigos de periódicos publicados pelos docentes vinculados a FM.

\section{Tabela 2}

Número de docentes pertencentes aos cursos de fonoaudiologia da USP credenciados a programas de pósgraduação.

\begin{tabular}{lcccc}
\hline Unidade & $\begin{array}{c}\text { Número de } \\
\text { Docentes }\end{array}$ & $\begin{array}{c}\text { Docentes credenciados } \\
\text { na Pós-Graduação }\end{array}$ & $\begin{array}{c}\text { Números de programas } \\
\text { de Pós-Graduação }\end{array}$ & $\begin{array}{c}\text { Número de artigos } \\
\text { analisados }\end{array}$ \\
\hline FMRP & 7 & 4 & 1 & 87 \\
FM & 13 & 12 & 8 & 453 \\
FOB & 24 & 14 & 18 & 508 \\
\hline
\end{tabular}

\section{Tabela 3}

Média dos artigos na Plataforma Lattes, artigos indexados no ISI e o índice-h dos docentes de cada unidade dos cursos graduação em fonoaudiologia da Universidade de São Paulo.

\begin{tabular}{lccc}
\hline Unidade & Artigos na Plataforma Lattes & Artigos indexados no ISI & Índice $\mathrm{h}$ \\
\hline FMRP & 13,71 & 3 & 0,83 \\
FM & 34,92 & 2,77 & 0,85 \\
FOB & 21 & 2,58 & 0,71 \\
\hline
\end{tabular}

graduação em fonoaudiologia foram encontradas nas análises de dados. Os resultados a partir do Currículo Lattes de cada docente, em geral, apresentaram uma alta concentração de publicação em algumas revistas.

A publicação dos artigos advindos dos docen- tes da FM concentraram-se $40 \%$ em duas revistas: Pró-Fono e Revista da Sociedade Brasileira de Fonoaudiologia. Já na FOB, foi possível observar que $30 \%$ dos artigos são publicados em três revistas: Revista Brasileira de Otorrinolaringologia, Revista da Sociedade Brasileira de Fonoaudiologia e revista Pró- 
Fono. Na FMRP houve uma homogeneidade maior, na qual $50 \%$ dos artigos foram publicados em 6 revistas. Vale ressaltar que estas revistas com quantidade elevada de artigos publicados, fazem parte do grupo de revistas de fonoaudiologia e áreas afins (otorrinolaringologia) de repercussão e/ou prestígio nacional. Possivelmente, isto explique a alta concentração de publicações em poucos periódicos.

Foram encontrados em números absolutos, na plataforma Lattes, menos referências de artigos dos docentes pertencentes à FMRP. Isto pode ter ocorrido devido ao pouco tempo de criação do curso de fonoaudiologia dessa unidade, se comparado a FOB e FM. Além disso, poucos docentes pertencentes à FMRP são credenciados em programas de pós-graduação.

Observou-se também que os docentes vinculados aos três cursos de graduação em fonoaudiologia da Universidade de São Paulo tiveram poucos artigos indexados ao ISI e baixos índices-h. De acordo com Torro-Alves et al ${ }^{11}$, o índice h é proporcional ao número de artigos indexados no ISI. Podemos atribuir parte desta constatação pela cobertura limitada do ISI $^{12-15}$. Isto ocorre porque apenas $5 \%$ dos artigos publicados no mundo estão incluídos no ISI.

A fonoaudiologia possui um campo de concentração do qual fazem parte cinco áreas: a linguagem, a motricidade orofacial, a voz, a audiologia e mais recentemente a saúde coletiva. Sendo assim, alguns trabalhos são de interesse locais, regionais ou nacionais, por exemplo, quando se estuda parâmetros de uma população específica (diferenças lingüísticas regionais, a linguagem de crianças de determinadas escolas, dentre outros). Dessa forma, os artigos têm maior impacto se forem publicados no Brasil ${ }^{11,12}$.

Mas por outro lado, muitos pesquisadores brasileiros em fonoaudiologia produzem trabalhos os quais mereçam e atendem a necessidade não só local/regional, mas sim mundial. Pesquisas na área reabilitação após colocação de implante coclear, oromiofaciais, disfluências, dentre outras, com excelente qualidade merecem ser apreciadas por fonoaudiólogos de todo o mundo. Muitos desses trabalhos não são enviados às revistas internacionais de excelência, assim diminuem as chances de serem reconhecidos e valorizados. Talvez neste caso, os pesquisadores devam acreditar mais na qualidade de seus trabalhos.

Se pesquisadores brasileiros são pagos com dinheiro nacional e têm suas pesquisas financiadas por órgãos de fomento brasileiro, suas pesquisas devem ser publicadas no Brasil para que essas sejam apreciadas pelos profissionais e atendam às necessidades da população brasileira? É claro que se o dinheiro investido é nacional, deve servir à população local. Porém, é possível atender essa população e também às necessidades mundiais (como acontece em outras ciências, como a física e biologia, por exemplo), pois muitas dessas são também necessidades brasileiras. Sem contar que com o advento da Internet, uma pessoa em qualquer lugar do mundo pode ter acesso à maioria das publicações, desta forma, estaria atendendo também os profissionais brasileiros.

Com a indexação do SciELO pelo SCOPUS, algumas revistas como Pró-Fono, Revista da Sociedade Brasileira de Fonoaudiologia, CEFAC e Revista Brasileira de Otorrinolaringologia já estão internacionalizadas ${ }^{16,17}$. Sendo assim, é provável que os trabalhos realizados por pesquisadores brasileiros possam ser apreciados por um número maior de leitores de todo o mundo, aumentando assim a contribuição da comunidade fonoaudiológica brasileira para a ciência mundial. Porém, é interessante se pensar na possibilidade de redigir tais revistas também na língua inglesa.

Foi observado que muitos artigos de fonoaudiologia no Brasil são publicados em periódicos não indexados no IS ${ }^{15}$. Desta forma, apenas a plataforma Thompson ISI Web of Science não pode ser considerada como parâmetro para medir a produtividade destes docentes. Considerando aspectos mais amplos, entende-se que o Currículo Lattes4 é uma importante ferramenta para medir a produtividade científica destes docentes, pois além de mostrar os artigos publicados, é possível observar as orientações concluídas e/ ou em andamento, os projetos de pesquisa concedidos pelas agências de fomento (CNPq, FINEP, CAPES, FAPESP) e etc.

\section{Conclusőes}

Em resumo, o atual trabalho demonstrou que os docentes dos três cursos de graduação em fonoaudiologia da Universidade de São Paulo têm a maioria de suas publicações concentradas em poucas revistas, tais como: Pró-Fono, Revista da Sociedade, Jornal Brasileiro de Fonoaudiologia, Brasileira de Fonoaudiologia e Revista Brasileira de Otorrinolaringologia, e estas não fazem parte do banco de dados ISI. Já a Plataforma Lattes apresenta outros conteúdos que podem ser considerados para mensurar a produtividade científica de tais pesquisadores. 


\section{ABSTRACT}

The University of São Paulo has three undergraduate courses of speech-language and hearing science offer by Faculty of Medicine (FM), Faculty of Odontology of Bauru (FOB) and Faculty of Medicine of Ribeirão Preto (FMRP). Using data retrieved from the Lattes Plataform database (Curriculum Lattes) we have quantitatively the scientific productivity percentage of speech, language \& hearing science professors at USP. We observed that bibliometric evaluations using the Curriculum Lattes $(\mathrm{CL})$ showed that the professors published papers in journal are not indexed by ISI. We showed that speech-language and hearing science professors at USP concentrated his paper in few journals (Brazilian Journal of Speech, Language \& Hearing, CEFAC, Pró-Fono, Journal of Brazilian Society of Speech, Language and Hearing Science and Brazilian Journal of Otorhinolaryngology).

Keywords: Periodical Index. Scientific Publication Indicators. Bibliometric Indicators.

\section{Referênclas}

1. Castro RCF. Impacto da internet no fluxo da comunicação científica em saúde. Rev Saúde Pública 2006; 40: 57-63

2. Pellegrini Filho A. Ciencia en pro de la salud: notas sobre la organización de la actividad científica para el desarrollo de la salud en América Latina y el Caribe. Washington (DC): Organización Panamericana de la Salud; 2000. [OPS Publicación Científica y Técnica, 578]

3. Price DJ. O desenvolvimento da cidadania: análises histórica, filosófica, sociológica e econômica.Tradução de Simão Mathias. Rio de Janeiro: Livros Teóricos e Científicos, 1976; 96. Título Original: Little Science, Big Science

4. CNPq (Conselho Nacional de Desenvolvimento Científico e Tecnológico) Plataforma Lattes. 2009. http://buscatextual. cnpq.br/buscatextual/index.jsp. [Acessado em 10 de Fevereiro de 2009]

5. ISI (Thompson ISI Web of Science), ISI Web of Knowledge. 2009. http://portal.isiknowledge.com/portal.cgi?DestApp =WOS\&Func=Frame. [Acessado em 03 de Março de 2009]

6. SCOPUS (Scopus-Elsevier). 2009. http:// www.scopus.com. [Acessado em 22 de Janeiro de 2009]

7. Garfield E. Quantitative analysis of the scientificliterature and its implications for science policymakingin latin america and the caribbean. Bulletin of the PanAmerican Health Organization. 1995; 29. 87-95

8. SciELO (Scientific Electronic Library Online). 2009. http:// www.scielo.org . [Acessado em 02 de Janeiro de 2009]
9. Freitas MTA. O pensamento de Vygotsky e Bakhtin no Brasil. 4a ed. Campinas,(SP): Papirus Editora;1999.

10. Hirsch JE. An index to quantify an individual's scientific research output. Proc Natl Acad Sci USA. 2005; 102: 1656972.

11. Torro-Alves N, Herculano RD, Terçariol CAS, Filho OK, Graeff CFO. Hirsch's index: a case study conducted at the Faculdade de Filosofia, Ciências e Letras de Ribeirão Preto, Universidade de São Paulo. Braz J Med Biol Res. 2007; 40: 1529-36.

12. Herculano RD, Norberto $A M Q$. Comparison of scientific bibliographic productivity in undergraduate courses of speech-language and hearing science at USP using the Hirsh index. J Appl Sci 2009; 9: 4095-8.

13. Roediger HL. The h-index in Science: A new measure of scholarly contribution. Observer 19 (4).http://www. psychologicalscience.org/observer/getArticle.cfm?id=1971.

14. Glänzel W. On the h-index - A mathematical approach to a new measure of publication activity and citation impact. Scientometrics 2006; 67: 315-21.

15. Herculano RD, Torro-Alves N, Terçariol CAS, Norberto AMQ, Graeff CFO. Produção científica na Faculdade de Filosofia, Ciências e Letras de Ribeirão Preto - Universidade de São Paulo: Aplicação do índice de Hirsch. Medicina (Ribeirão Preto). 2008 ; $41: 343-50$.

16. Strehl L, dos Santos CA. Indicadores de qualidade da atividade científica. Ciênc Hoje. 2002; 31: 34-9.

17. Meneghini R. The Internacionalization of Reports in Health Sciences. Cad Saúde Pública 2007; 23: 2260-1. 